were evaluated at T1 and T2 using self-report items elaborated from a national survey. Self-rated health status was assessed at both time points using one validated self-report item. Intervention exposure was measured by questionnaire and complemented by qualitative analyses.

Result Between T1 and T2, the prevalence of weekly physical activity frequency increased for men and the prevalence of exsmokers increased for both men and workers aged 55 or older. When body mass index was taken into account, the prevalence of daily fruit and vegetable consumption also increased for both men and workers aged 55 or older. However, these workers' self-reported health status remained unchanged from T1 to T2. No clear changes were observed in other age groups.

Discussion The improvement of lifestyle habits for both male workers and those aged 55 or older following HES interventions is significant since scientific literature suggests that those groups are generally less inclined to participate in health promotion workplace interventions.

\section{WHAT ARE GOOD JOBS? A EUROPEAN PEROSH PERSPECTIVE}

${ }^{1}$ David Fishwick*, ${ }^{1}$ Edward Robinson, ${ }^{2}$ Noortje Wiezer, ${ }^{3}$ Zofia Mockalo, ${ }^{4}$ Vincent Grosjean, ${ }^{5}$ Lars L Andersen. ${ }^{1}$ Centre for Workplace Health, HSE, Buxton, Derbyshire, UK; ${ }^{2} T N O$, Leiden, Netherlands; ${ }^{3} \mathrm{CIOP}$, Warsaw, Poland; ${ }^{4}$ Département Homme au Travail, INRS, France; ${ }^{5}$ National Research Centre for the Working Environment, Copenhagen, Denmark

\subsection{6/oemed-2018-ICOHabstracts. 1657}

Introduction PEROSH stands for the Partnership for European Research in Occupational Safety and Health. The work of this group relates to developing a European perspective concerning wellbeing, and importantly organising worker wellbeing conferences. Our most recent conference developed a 'Good Jobs' theme.

Methods Wellbeing experts participated in a knowledge café event, rotating between three semi-structured discussions relating to:

i. what makes a good job,

ii. roles of leadership and

iii. good jobs in harsh economic times.

Results What makes a good job; individual factors including autonomy, support, recognition, development through workplace learning programmes, optimum work-life balance, adaptation to different working life phases, adequate financial rewards and the ability to focus on primary task were identified as important. The nature of the work was felt important to result in engagement and challenge, be safe and inclusive, and both be meaningful and personally flexible.

Leadership roles; associated with good jobs included the ability to creatively motivate and engage workers, provide social support, coaching, mentoring and empathy, and foster a good balance between shareholder and stakeholder requirements. Participation, instead of command and control, appeared key, as did recognition and management of interpersonal conflicts at work. Leaders were favoured who allowed autonomy, facilitated development and progress, and were seen as role models.

Good jobs in harsh economic times; were identified to require many individual attributes centred around purposeful individual rewards, work attributes that considered issues that did not cost money to improve (e.g. better communication around change), and work site attributes centred around investing in people as important resources and innovative labour relations.

Discussion PEROSH has successfully developed a view relating to what constitutes a good job. It is hoped that the output from this process will help shape the future workplace and employment policies of each of the represented European countries.

\section{WORKPLACE BULLYING AND METABOLIC SYNDROME AMONG PRIVATE SECTOR WORKFORCE IN MALAYSIA}

${ }^{1}$ VYW Lai, ${ }^{1}$ AT Su, ${ }^{2} \mathrm{M}$ Isahak, ${ }^{2}$ VCW Hoe, ${ }^{3}$ SSM Kwan, ${ }^{4} \mathrm{~A}$ Darus, ${ }^{5} \mathrm{~K}$ Tsuno. ${ }^{1}$ Universiti Malaysia Sarawak, Kota Samarahan, Malaysia; ${ }^{2}$ University of Malaya, Kuala Lumpur, Malaysia; ${ }^{3}$ University Malaysia Sabah, Kota Kinabalu, Malaysia; ${ }^{4}$ Social Security Organisation of Malaysia (SOCSO), Kuala Lumpur, Malaysia; ${ }^{5}$ Wakayama Medical University, Wakayama, Japan

\subsection{6/oemed-2018-ICOHabstracts. 1658}

Introduction Workplace bullying is increasingly recognised as a serious public health issue in the workplace because of its increasing prevalence worldwide and negative health impacts on employee's health. The consequences vary from stress and depression to psychosomatic problems including cardiovascular diseases. The objective of this study is to determine the prevalence of workplace bullying and its association with metabolic syndrome among private industry workers in Malaysia.

Methods Workers undergoing Health Screening Program (HSP) by Social Security Organisation Malaysia in a private healthcare laboratory throughout Malaysia were recruited into the study. The targeted sample size was 1580 participants throughout the country. Workplace bullying was measured using Modified Negative Acts Questionnaire and Malaysian Workplace Bullying Index. Data collected from the HSP was analysed to determine the presence of metabolic syndrome. The relationship between the presence of workplace bullying and metabolic syndrome was analysed using SPSS software.

Results Preliminary results showed most of the respondents experienced more on workrelated bullying than person-related bullying. Age and waist circumference were significantly associated with workplace bullying. There was no significant association between workplace bullying and other parameters of metabolic syndrome. Data collection is still in progress and will be completed by February 2018 .

Conclusion The prevalence of workplace bullying is related to age and possible obesity. Its association with metabolic syndrome is inconclusive for the time being.

\section{INFLUENCE OF WORK ORGANISATION AND ENVIRONMENT ON HEALTH BEHAVIOURS OF CONSTRUCTION APPRENTICES}

1,2BA Evanoff*, $2,3 \mathrm{D}$ Rohlman, ${ }^{1,2}$ JR Strickland, ${ }^{2,3} \mathrm{KM}$ Kelly, ${ }^{1,2} \mathrm{AM}$ Dale. ${ }^{1}$ Division of
General Medical Sciences, Washington University School of Medicine in St. Louis, St. Louis,
MO, USA; ${ }^{2}$ Healthier Workforce Centre of the Midwest, lowa City, IA, USA; ${ }^{3}$ College of
Public Health, University of lowa, lowa City, IA, USA

10.1136/oemed-2018-ICOHabstracts. 1659

Introduction Construction is among the most dangerous industries with well-recognised high physical demands and low job autonomy. In addition to traditional hazards for workplace injury and illness, other threats to health and well-being occur 
from work organisation and work environment factors, including irregular employment, multiple job sites, long commutes, long work hours, and employer policies regarding health and safety. These non-traditional hazards are associated with injury and illness, as well as health behaviours including poor diet, smoking, and psychosocial stress. The cumulative impact of both traditional and non-traditional hazards on the health and well-being of construction workers are largely unknown.

Methods We conducted a survey among a cohort of apprentice construction workers to identify relationships between work organisation, environmental factors, health behaviours, and health outcomes.

Results 960 surveys have been completed to date. Preliminary analyses show that respondents (mean age 28) report high levels of job satisfaction, job security, and social support, but also report high rates of musculoskeletal symptoms and various work organisational factors potentially affecting health and health behaviours. Average commuting distance to work is over $60 \mathrm{~km}$, and $63 \%$ report no limits on daily working hours. Despite high smoking rates (28\% are current smokers), only 55\% report any restrictions on smoking at their worksites. Only $10 \%$ report regularly using sunscreen when in the sun for >15 min; only 4\% of worksites provided sunscreen.

Discussion These preliminary results highlight non-traditional worksite health risks, and suggest potential interventions that may reduce the high rates of risky heath behaviours among construction workers. As our study progresses, we plan to evaluate the impact of work organisation on health and health behaviours in three construction trades; identify workplace programs, policies, and practices affecting worker health and well-being; and determine readiness for adoption of integrated interventions to improve worker health.

\section{WORKPLACE PRACTICES AND POLICIES TO SUPPORT WORKERS WITH DEPRESSION}

D Van Eerd*, K Cullen, E Irvin, MLe Pouésard. Institute for Work and Health, Toronto, Canada

\subsection{6/oemed-2018-ICOHabstracts. 1660}

Introduction The burden associated with the effects of depression in the workplace is extensive. Workers with depression lose more health-related productive time, have higher rates of absenteeism and short-term disability, and experience higher rates of job turnover than those without depression. Our objective is to synthesise evidence from the scientific literature, practice evidence (workplace policies and practices), and experiences from OHS stakeholders.

Methods Our study sample is OHS stakeholders: workers, managers, and consultants in workplaces from our contact database (approximately 700 OHS contacts across Canada willing to be contacted for research). Data collection includes a web-based survey, focus groups (ongoing), and interviews (ongoing) with stakeholder representatives from multiple sectors. We are collecting information about workplace practices and policies to prevent productivity losses, promote stay-atwork, and support return-to-work for workers with depression. The synthesis is a two stage process: first synthesising practice evidence gathered from stakeholders and then combining that with evidence from the scientific literature.

Results Preliminary results ( $n=400,73 \%$ workers, 27\% managers/consultants) reveal a willingness among participants to share their experiences with depression and work. Workers report practices related to non-judgemental listening and external supports were most helpful to them. Managers/consultants suggest non-judgemental listening and employee assistance programs were most helpful. However, workers often feel workplace support is lacking and report non-supportive supervisors as a key barrier to receiving needed support. Managers indicate a lack of training and knowledge about depression are the main barriers to providing support to workers. Workers did not feel providing information was helpful whereas managers often did.

Conclusion Preliminary results reveal the importance of nonjudgemental listening as a workplace support for depression. However, responses reveal workers and managers do not have similar experiences of workplace support. Synthesising practice and scientific evidence will help guide policies and practices to support workers with depression.

\section{PREVENTING WORK DISABILITY IN WORKERS WITH DEPRESSION; A SYSTEMATIC REVIEW}

K Cullen* , E Inin, D Van Eerd, R Saunders. Institute for Work and Health, Toronto, Canada

\subsection{6/oemed-2018-ICOHabstracts. 1661}

Introduction By the year 2020, depression will be the second most burdensome illness in developed economies. In addition to its adverse individual effects, the associated workplace effects of depression are extensive. This review is provides a synthesis of the evidence to determine effective intervention approaches for managing depression in the workplace: both to help workers stay-at-work while experiencing symptoms and to return-to-work after an episode of time away from work.

Methods We followed a systematic review process developed by the Institute for Work and Health and an adapted best evidence synthesis. Articles that met the following criteria were considered: working age individuals with depression; workplace-based interventions; including a comparison group; outcomes of work functioning, work disability, or recurrences of work disability.

Result Seven electronic databases were searched from inception up to June 2015. The review examined 8123 titles and abstracts for relevance and found 20 RCTs and seven nRCTs from various jurisdictions. These studies evaluated a range of interventions, including; cognitive-behavioural therapy (CBT), work-focused CBT, problem solving therapy (PST), workfocused PST, enhanced care delivery, coordination of services, short- and long-term psychodynamic therapy, stress reduction programs, exercise training, part-time sick leave and naturebased rehabilitation. Our findings indicate that CBT and PST interventions can help workers with depression stay-at-work while managing their symptoms; however, only work-focused CBT is sufficient to help workers return-to-work after an absence. There is currently not enough evidence from the scientific literature to guide practice for the remaining interventions identified in this review.

Discussion We synthesised the current best evidence on workplace interventions to help employees manage symptoms of depression. The interventions identified in this review focused on strategies targeting personal resilience and coping skills of individuals with depression. More work is needed to evaluate interventions aimed at mitigating workplace factors such as psychosocial working conditions. 